\title{
Statistical Thermodynamics of Polymer Quantum Systems
}

\author{
Guillermo CHACÓN-ACOSTA ${ }^{\dagger}$, Elisa MANRIQUE ${ }^{\ddagger}$, Leonardo DAGDUG ${ }^{\S}$ \\ and Hugo A. MORALES-TÉCOTL $§$
}

† Departamento de Matemáticas Aplicadas y Sistemas, Universidad Autónoma Metropolitana-Cuajimalpa, Artificios 40, México D. F. 01120, México

E-mail: gchacon@correo.cua.uam.mx

$\ddagger$ Institut für Physik, Johannes-Gutenberg-Universität, D-55099 Mainz, Germany

E-mail: manrique@zino.physik.uni-mainz.de

$\S$ Departamento de Física, Universidad Autónoma Metropolitana-Iztapalapa, San Rafael Atlixco 186, México D. F. 09340, México

E-mail:dll@xanum.uam.mx,hugo@xanum.uam.mx

Received September 01, 2011, in final form November 16, 2011; Published online December 02, 2011 http://dx.doi.org/10.3842/SIGMA.2011.110

\begin{abstract}
Polymer quantum systems are mechanical models quantized similarly as loop quantum gravity. It is actually in quantizing gravity that the polymer term holds proper as the quantum geometry excitations yield a reminiscent of a polymer material. In such an approach both non-singular cosmological models and a microscopic basis for the entropy of some black holes have arisen. Also important physical questions for these systems involve thermodynamics. With this motivation, in this work, we study the statistical thermodynamics of two one dimensional polymer quantum systems: an ensemble of oscillators that describe a solid and a bunch of non-interacting particles in a box, which thus form an ideal gas. We first study the spectra of these polymer systems. It turns out useful for the analysis to consider the length scale required by the quantization and which we shall refer to as polymer length. The dynamics of the polymer oscillator can be given the form of that for the standard quantum pendulum. Depending on the dominance of the polymer length we can distinguish two regimes: vibrational and rotational. The first occur for small polymer length and here the standard oscillator in Schrödinger quantization is recovered at leading order. The second one, for large polymer length, features dominant polymer effects. In the case of the polymer particles in the box, a bounded and oscillating spectrum that presents a band structure and a Brillouin zone is found. The thermodynamical quantities calculated with these spectra have corrections with respect to standard ones and they depend on the polymer length. When the polymer length is small such corrections resemble those coming from the phenomenological generalized uncertainty relation approach based on the idea of the existence of a minimal length. For generic polymer length, thermodynamics of both systems present an anomalous peak in their heat capacity $C_{V}$. In the case of the polymer oscillators this peak separates the vibrational and rotational regimes, while in the ideal polymer gas it reflects the band structure which allows the existence of negative temperatures.
\end{abstract}

Key words: statistical thermodynamics; canonical quantization; loop quantum gravity

2010 Mathematics Subject Classification: 82B30; 81S05; 81Q65; 82B20; 83C45

\footnotetext{
*This paper is a contribution to the Special Issue "Loop Quantum Gravity and Cosmology". The full collection is available at http://www.emis.de/journals/SIGMA/LQGC.html
} 


\section{Introduction}

In coping with the challenge of quantizing gravity the loop quantization approach [1, 2] has proved convenient to incorporate the background independent character demanded by general relativity. Important progress in this approach include the avoidance of the classical singularity which in loop quantum cosmology is replaced by a quantum bounce [3] in physically motivated models, $[4,5,6]$, and a microscopic basis for the entropy of some black holes that is in accordance with the Bekenstein-Hawking's semiclassical formula [7, 8, 9].

However some further physical questions in regard to cosmology and black holes necessarily involve thermodynamics. As it is well known primordial particle backgrounds including neutrinos, gravitons and photons, originating in the early universe provide windows to explore such early stage $[10,11]$. For instance the stochastic graviton remnant and its statistical properties have been studied [12, 13]. Also the different contributions to the spectrum of gravitons produced during the super-inflationary period have been investigated [14, 15, 16, 17]. In regard to black holes, from the loop quantum gravity perspective, the challenge remains of describing black hole evaporation including in particular its thermodynamical aspects (see e.g. [18] for recent work.)

Rather than dealing with the thermodynamics of loop quantized gravitational systems a more tractable problem is to consider the statistical thermodynamics of polymer quantum systems $[19,20,21]$. The latter are mechanical systems quantized following loop quantum gravity. Here an important comment in regard to the polymer term is in order. In the gravitational case, loops, or more strictly, graphs, label quantum states of gravity. This yields a picture resembling polymer materials, which justifies adopting the term polymer quantization for gravity. However, as we will see below, for mechanical systems states will be labeled by point sets belonging to a lattice. Thus, although the term polymer is inherited from the gravity case it is not actually realized in the mechanical one. We should stress at this point also that in loop or polymer quantization a length scale is required for its construction, while for the gravitational case this is identified with Planck's length, in the mechanical case it is just a free parameter and we refer to it as the polymer length scale. It should be mentioned though that, technically, the quantization here dubbed polymer, was previously considered from a different perspective in the form of a non regular representation of the canonical commutation relation [22]. Interestingly, a quantization based on difference operators has also been considered [23].

Polymer quantum systems have been convenient to illustrate some features arising in loop quantum gravity $[19,24]$. In particular they have the same configuration space as that of loop quantum cosmology [25]. The continuum limit of the polymer quantum system has also been explored using ad hoc renormalization schemes in which the polymer length scale runs [20, 21]. Investigating whether polymer quantum system admits Galilean symmetry has been reported in [26]. Inspired by the cosmic singularity avoidance this quantization has been used to explore potentials such as $1 / r$ [27], and $1 / r^{2}$ [28].

In this work we shall study the thermostatistics of two simple polymer systems, namely, an ensemble of oscillators and a bunch of noninteracting particles in a box. The paper is structured as follows. In Section 2 we review the basics of polymer quantization of mechanical systems, in particular the eigenvalue problem. For the harmonic oscillator the corresponding eigenvalue problem can be casted in Fourier space as a second order differential equation. In this way one can see its spectrum is just that of the standard quantum pendulum. Two regimes can be seen to appear: an oscillatory or vibrational one and a rotational one. In the first, the oscillator in Schrödinger quantization is recovered, at leading order, while in the second regime, the polymer effects are dominant. Furthermore, we solve exactly the eigenvalue problem for the polymer particle in a box that takes the form of a second order difference equation. We obtain a bounded and oscillating spectrum that features a band structure and a Brillouin zone.

In Section 3 we calculate the corresponding thermodynamical quantities with these spectra. They depend on the polymer length and behave differently from those in standard thermo- 
dynamics, which is recovered when the polymer length is considered small. In this case the thermodynamical variables can be written as a series on the small polymer length, similarly to what happens in the phenomenological Generalized Uncertainty Principle (GUP) approach based on the idea of the existence of a minimal length $[29,30]$. These were applied previously to an ideal gas [31, 32, 33], and radiation [34, 35]. (The modification to the statistical mechanics of systems were also studied from the perspective of the extension to the Standard Model that have Lorentz violating terms [36], and the case of radiation was also studied with corrections arising from loop quantum gravity [37,38].) In some sense this behavior could be expected since the uncertainty relation for polymer systems is quite similar to GUP [19, 39]. We shall show quadratic corrections occur in the energy of the polymer particle in a box, just as in GUP, but with opposite sign. For a generic, not necessarily small, polymer length, thermodynamics of both systems present an anomalous peak in their heat capacity $C_{V}$. For the polymer oscillators this peak separates the vibrational and rotational regimes, while in the ideal polymer gas it reflects the band structure. It is worth stressing that, for the gas, the band structure allows for the existence of negative temperatures.

Finally in Section 4 we discuss our results and point out some perspectives.

\section{Eigenvalue problem in the polymer representation of quantum mechanics}

In this section we describe the main features of the polymer quantization of a non relativistic particle moving on the real line $[19,20]$, and describe briefly the corresponding eigenvalue problem. To do so we start by noticing that instead of the Heisenberg algebra, involving position $\hat{q}$ and momentum $\hat{p}$ of the particle

$$
[\hat{q}, \hat{q}]=[\hat{p}, \hat{p}]=0, \quad[\hat{q}, \hat{p}]=\hat{q} \hat{p}-\hat{p} \hat{q}=i \hat{I},
$$

where $\hat{I}$ is the identity on Hilbert space $\mathcal{H}$, one adopts the Weyl algebra

$$
\begin{aligned}
& U\left(\lambda_{1}\right) \cdot U\left(\lambda_{2}\right)=U\left(\lambda_{1}+\lambda_{2}\right), \quad V\left(\mu_{1}\right) \cdot V\left(\mu_{2}\right)=V\left(\mu_{1}+\mu_{2}\right), \\
& \hat{U}(\lambda) \cdot \hat{V}(\mu)=e^{-i \lambda \mu} \hat{V}(\mu) \cdot \hat{U}(\lambda) .
\end{aligned}
$$

According to Stone-von Neumann theorem the elements of the above algebras can be related under certain conditions, including in particular weak continuity. Namely

$$
\hat{U}(\lambda)=e^{i \lambda \hat{q}}, \quad \hat{V}(\mu)=e^{i \mu \hat{p}},
$$

which however, does not hold in the polymer case.

In the usual or Schrödinger representation of quantum mechanics the Hilbert space is $\mathcal{H}=$ $L^{2}(\mathbb{R}, d x)$ with the Lebesgue measure $d x$. Instead, in the loop or polymer representation the kinematical Hilbert space $\mathcal{H}_{\text {poly }}$ is the Cauchy completion of the set of linear combination of some basis states $\left\{\left|x_{j}\right\rangle\right\}$, whose coefficients have a suitable fall-off [19], and with the following inner product

$$
\left\langle x_{i} \mid x_{j}\right\rangle=\lim _{T \rightarrow \infty} \frac{1}{T} \int_{a}^{a+T} d k e^{i k\left(x_{i}-x_{j}\right)}=\delta_{x_{i}, x_{j}},
$$

where $\delta_{x_{i}, x_{j}}$ is the Kronecker delta, instead of Dirac delta as in Schrödinger representation, then we say that the orthonormal basis is discrete. The kinematical Hilbert space can be written as $\mathcal{H}_{\text {poly }}=L^{2}\left(\mathbb{R}_{d}, \mathrm{~d} \mu_{d}\right)$ with $d \mu_{d}$ the corresponding Haar measure, and $\mathbb{R}_{d}$ the real line endowed with the discrete topology ${ }^{1}$.

\footnotetext{
${ }^{1}$ In the momentum representation, the configuration space is the Bohr compactification of the real line $\mathbb{R}_{B}$, $[19,25]$.
} 
As we already notice Stone-von Neumann's theorem is no longer applicable since the operator $V(\mu)$ fails to be weakly continuous on the parameter $\mu$ due to the discrete structure assigned to space [40, 19]. The shift operator $\hat{V}(\mu)$ is not related to any Hermitian operator as infinitesimal generator. Hence, for the representation of the Weyl algebra we choose the position operator $\hat{x}$ and the translation $\hat{V}(\mu)$ instead of the momentum operator. Its action on the basis is:

$$
\hat{x}\left|x_{j}\right\rangle=x_{j}\left|x_{j}\right\rangle, \quad \hat{V}(\mu)\left|x_{j}\right\rangle=\left|x_{j}-\mu\right\rangle,
$$

fulfilling also:

$$
[\hat{x}, \hat{V}(\mu)]=-\mu \hat{V}(\mu)
$$

Since there is no well defined momentum operator, any function on phase space which depends on the momentum has to be regularized. In particular this is so for the Hamiltonian. To do so we introduce an extra structure, namely a regular lattice with spacing length $\mu_{0}$. This analogue of what happens in Loop Quantum Cosmology, where there is a fundamental minimum area $[3,4,5,6]$ given in terms of Planck length.

Let us consider $\mu_{0}>0$ as any fixed scale. In general $\mu_{0}$ can be function of $x$ but here we suppose it is constant. One of the simplest options to define an operator analogous to the momentum operator is:

$$
\hat{K}_{\mu_{0}}=\frac{1}{2 i \mu_{0}}\left(\hat{V}\left(\mu_{0}\right)-\hat{V}\left(-\mu_{0}\right)\right) .
$$

This choice can be thought of as the formal replacement

$$
\hat{p} \rightarrow \frac{1}{\mu_{0}} \widehat{\sin \left(\mu_{0} p\right)},
$$

where the right hand side is given by (2.1). Some authors have studied a semiclassical regime in which the expectation value of the Hamiltonian is taken with respect to a semiclassical state to yield an effective dynamics that can be seen, at leading order, as obtained from the replacement $p \rightarrow \sin \left(\mu_{0} p\right) / \mu_{0}$ in the classical Hamiltonian. This has been proved useful in several models $[41,42,43,44,45]$.

Thus the polymer Hamiltonian is written as

$$
\widehat{H}_{\mu_{0}}=\frac{\hbar^{2}}{2 m \mu_{0}^{2}}\left[2-\hat{V}\left(\mu_{0}\right)-\hat{V}\left(-\mu_{0}\right)\right]+\hat{W}(x),
$$

where $W(x)$ is a potential term. The dynamics generated by (2.2) decomposes the polymer Hilbert space $\mathcal{H}_{\text {poly }}$, into an infinite superselected finite-dimensional subspaces, each with support on a regular lattice $\gamma=\gamma\left(\mu_{0}, x_{0}\right)$ with the same space between points $\mu_{0}$, where $\gamma\left(\mu_{0}, x_{0}\right)=\left\{n \mu_{0}+x_{0} \mid n \in \mathbb{Z}\right\}$, and $x_{0} \in\left[0, \mu_{0}\right)$. Thus, choosing $x_{0}$ fixes the superselected sector.

There are at least two possible ways to regain Schrödinger quantum mechanics. One is to consider the polymer length scale $\mu_{0}$ as small such that the difference equation (2.2) can be approximated by the usual differential Schrödinger equation [19]. Another possibility is to consider the introduction of the lattice as an intermediate step, after which it is necessary to carry out a renormalization procedure, as it is usually done in lattice theories [46]. In polymer quantization the final result turns out to be the usual Schrödinger quantum mechanics [21, 20].

Next we consider the eigenvalue problem:

$$
\widehat{H}_{\mu_{0}}|\psi\rangle=E|\psi\rangle \text {. }
$$


In the case of the Hamiltonian (2.2) on the lattice $\gamma\left(x_{0}, \mu_{0}\right)$, any state $|\psi\rangle \in \mathcal{H}_{\text {poly }}$ is of the form:

$$
|\psi\rangle=\sum_{j \in \mathbb{Z}} \psi\left(x_{0}+j \mu_{0}\right)\left|x_{0}+j \mu_{0}\right\rangle
$$

The substitution of (2.4) into (2.3) gives a difference equation in the position representation

$$
\frac{\hbar^{2}}{2 m \mu_{0}^{2}}\left[2 \psi\left(x_{j}\right)-\psi\left(x_{j}+\mu_{0}\right)-\psi\left(x_{j}-\mu_{0}\right)\right]=\left[E-W\left(x_{j}\right)\right] \psi\left(x_{j}\right) .
$$

Using the Fourier transform defined by

$$
\psi(k)=\left(k|\psi\rangle=\sum_{j \in \mathbb{Z}} \psi\left(x_{0}+j \mu_{0}\right) e^{-i k\left(x_{0}+j \mu_{0}\right)},\right.
$$

where $k \in\left[-\pi / \mu_{0}, \pi / \mu_{0}\right]$ and with $\psi(k)$ satisfying the condition

$$
\psi\left(\frac{\pi}{\mu_{0}}\right)=e^{-2 \pi i \frac{x_{0}}{\mu_{0}}} \psi\left(-\frac{\pi}{\mu_{0}}\right)
$$

the equation (2.5) reads:

$$
\left(1-\frac{\mu_{0}^{2} m}{\hbar^{2}} E-\cos k \mu_{0}\right) \psi(k)=-\frac{\mu_{0}^{2} m}{\hbar^{2}} \sum_{j \in \mathbb{Z}} \psi\left(x_{0}+j \mu_{0}\right) W\left(x_{0}+j \mu_{0}\right) e^{-i k\left(x_{0}+j \mu_{0}\right)} .
$$

In our analysis two specific cases will be considered: the harmonic oscillator and the particle in a box.

\section{$2.1 \quad$ Harmonic oscillator}

The polymer harmonic oscillator has been already studied in [19] and [47] ${ }^{2}$, using the potential $W(x)=\hbar \omega x^{2} / 2 d^{2}$, with $d^{2}=\hbar / m \omega$ a characteristic length of the oscillator. After performing the Fourier transform in the r.h.s. of (2.6) and using the quadratic potential, one obtains a second order differential equation which can be recognized as a Mathieu equation [49]

$$
\frac{d^{2} \psi(\phi)}{d \phi^{2}}+(a-2 q \cos 2 \phi) \psi(\phi)=0
$$

where $\phi=\frac{k \mu_{0}+\pi}{2}, a=\frac{8}{\lambda^{4}}\left(\frac{\lambda^{2}}{\hbar \omega} E-1\right)$ and $q=4 \lambda^{-4}$, and we introduce $\lambda:=\mu_{0} / d$ as a dimensionless length parameter. This system is just a quantum pendulum in $k$ space [20]. For low energies one recovers the harmonic oscillator behavior, while for high energies it becomes a free rigid rotor [50]. Equation (2.7) has periodic solutions for particular values of $a=a_{n}, b_{n}$ called the Mathieu characteristic functions, that depend on $n,[49]$. The corresponding wave functions can be written in terms of the Mathieu elliptic sine and cosine [47]. The energy eigenvalues can be expressed as follows $[49,47]$

$$
\begin{aligned}
& E_{2 n}=\frac{\hbar \omega}{\lambda^{2}}\left[1+\frac{\lambda^{4}}{8} a_{n}\left(\frac{4}{\lambda^{4}}\right)\right], \\
& E_{2 n+1}=\frac{\hbar \omega}{\lambda^{2}}\left[1+\frac{\lambda^{4}}{8} b_{n+1}\left(\frac{4}{\lambda^{4}}\right)\right] .
\end{aligned}
$$

\footnotetext{
${ }^{2}$ Actually a previous study appears in [48], in a different context, transport of electrons in semiconductors.
} 
An asymptotic expansion for the characteristic functions, assuming $\mu_{0} \ll d$ yields that the energy spectrum $E_{2 n} \approx E_{2 n+1}$, can be approximated as [19, 47]

$$
E_{n}=\left(n+\frac{1}{2}\right) \hbar \omega-\left(\frac{2 n^{2}+2 n+1}{32}\right) \lambda^{2} \hbar \omega+\mathcal{O}\left(\lambda^{4}\right) .
$$

As expected the spectrum consists of the standard harmonic oscillator plus corrections of order $\mathcal{O}\left(\lambda^{2}\right)$. However, this spectrum is not bounded from below. To enforce correspondence with the Schrödinger quantization, we can ask that the leading term be larger than the first correction. This gives us a maximum $n_{\max }$ that depends on $\lambda$,

$$
n_{\max } \approx \lambda^{-2}
$$

This means that, for small $\lambda$, we can not probe the spectrum with values of $n$ greater than those allowed by (2.11). As in [19] with the values of $\mu_{0}=10^{-19} \mathrm{~m}$, corresponding to the maximum experimental attainable energy today, and with $d=10^{-12} \mathrm{~m}$ for the carbon monoxide molecule, one gets $\lambda=10^{-7}$. In this case $n_{\max } \simeq 10^{14}$. This is consistent with [21] and [20] where a cut-off in the energy eigenvalues was introduced that depends on the regulator scale in order to perform the renormalization procedure that is necessary to implement a continuum limit of the theory.

On the opposite limit for $\lambda \gg 1$ the eigenstates are $E_{2 n} \approx E_{2 n-1}$, for $n=1,2, \ldots$.

$$
E_{n}=\frac{\hbar \omega}{\lambda^{2}}+\hbar \omega \frac{\lambda^{2}}{8} n^{2}+\mathcal{O}\left(\lambda^{-6}\right)
$$

where the ground state depends only on $\lambda^{-2}$, and actually falls off as $\lambda$ increases. As pointed out in [47] this case may be relevant for the cosmological constant problem. For the excited states $n \neq 0$, the $\lambda^{2}$ term dominates. In this regimen it is better to interpret $\lambda$ as a ratio of energies. Indeed the dimensionless parameter $\lambda^{2}=\frac{E_{\mathrm{osc}}}{E_{\mathrm{poly}}}$, where $E_{\mathrm{osc}}=\hbar \omega$ and $E_{\mathrm{poly}}=\frac{\hbar^{2}}{m \mu_{0}^{2}}$ that corresponds to the coefficient of the kinetic term in (2.5). Then this regime is the case of $E_{\text {osc }} \gg E_{\text {poly }}$; if we consider the polymer length to be the Planck length then this case corresponds to the trans-planckian region relevant for both inflationary cosmology and black holes [51].

Following the comparison with the quantum rigid rotor [52], we recall that the spectrum of a quantum rigid rotor in two dimensions is $\frac{n^{2} \hbar^{2}}{2 I}$, with $I=\frac{m R^{2}}{2}$ being the moment of inertia and $R$ the radius of the rotor. Notice that the second term in (2.12) has the same functional dependence on $n$ as for the rotor with effective moment of inertia $I_{\text {eff }}=\frac{(2 \hbar / \omega)^{2}}{m \mu_{0}^{2}}$ and $R_{\text {eff }}=\frac{2 \sqrt{2}}{\lambda} d$.

\subsection{Particle in a box}

The next example is a particle confined in a box of size $L=N \mu_{0}$. The free polymer particle was first studied in [20]. Here however we confine it to a box. In this case, instead of working in Fourier space we can use directly the difference equation (2.5). As in the standard case the potential is defined as

$$
W\left(x_{j}\right)= \begin{cases}0, & x_{0}<x_{j}<x_{0}+L, \\ \infty, & \text { otherwise }\end{cases}
$$

where $x_{0} \in\left[0, \mu_{0}\right)$. This potential is then realized through appropriate boundary conditions over elements of $\mathcal{H}_{\text {poly }}$. The particle behaves as a free particle inside the box and vanishes outside, namely

$$
\psi\left(x_{0}\right)=\psi\left(L+x_{0}\right)=0, \quad \forall x_{0} \in\left[0, \mu_{0}\right) .
$$


We can conveniently rewrite equation (2.5) by using $x_{j}=x_{0}+j \mu_{0}$, as

$$
\psi(j+2)-\left(2-\frac{2 m E \mu_{0}^{2}}{\hbar^{2}}\right) \psi(j+1)+\psi(j)=0 .
$$

Following [53], we propose the solution of the difference equation of second order (2.15), to be

$$
\psi(j)=a_{1} r_{1}^{j}+a_{2} r_{2}^{j},
$$

where $a_{i}$ are constants coefficients and $r_{i}$ are the roots of the characteristic equation:

$$
r^{2}-\left(2-\frac{2 m E \mu_{0}^{2}}{\hbar^{2}}\right) r+1=0
$$

The solutions of (2.17) are

$$
r_{ \pm}=\left(1-\frac{m E \mu_{0}^{2}}{\hbar^{2}}\right) \pm \frac{1}{2} \sqrt{\frac{8 m E \mu_{0}^{2}}{\hbar^{2}}\left(\frac{m E \mu_{0}^{2}}{2 \hbar^{2}}-1\right)} .
$$

For positive energies, the argument of the square root gives us a relation between energy and $2 \hbar^{2} / m \mu_{0}^{2}$. If $E \geq 2 \hbar^{2} / m \mu_{0}^{2}$, then the roots $r_{ \pm}$are real numbers ${ }^{3}$, but incompatible with the boundary conditions, therefore they yield the trivial solution $\psi\left(x_{j}\right)=0$. This leads to the idea that the minimum length scale $\mu_{0}$ imposes a cut-off on the energy, and hence, energies greater than the cut-off are unphysical. Thus, the only meaningful physical case is when $E<2 \hbar^{2} / m \mu_{0}^{2}$ which gives us complex roots for $r_{ \pm}$. The solution is given by:

$$
\psi(j)=C_{1} \sin (j \theta)+C_{2} \cos (j \theta),
$$

which is a parametrization of (2.16) in polar coordinates,

$$
\cos \theta=\left(1-\frac{m E \mu_{0}^{2}}{\hbar^{2}}\right), \quad \sin \theta=\frac{1}{2} \sqrt{\frac{8 m E \mu_{0}^{2}}{\hbar^{2}}\left(\frac{m E \mu_{0}^{2}}{2 \hbar^{2}}-1\right)} .
$$

Imposing the boundary conditions (2.14) in (2.18) we find that $\theta=n \pi \lambda$. In this case $\lambda=$ $\mu_{0} / L=1 / N$ and $n \in \mathbb{Z}$. The eigenfunction of (2.15) turns out to be

$$
\psi_{n}\left(x_{j}\right)=C \sin \left(n \pi \lambda \frac{x_{j}}{\mu_{0}}\right)=C \sin \left(n \pi \frac{j}{N}\right), \quad 0<j<N,
$$

where $C$ is a normalization factor

$$
C=\left[\sum_{j=0}^{N} \sin ^{2}(n \pi \lambda j)\right]^{-\frac{1}{2}}=\sqrt{\frac{2}{N}}
$$

With (2.19) we can write the eigenstate (2.4) as

$$
\left|\psi_{n}\right\rangle=\sqrt{\frac{2}{N}} \sum_{x_{j}} \sin \left(n \pi \lambda \frac{x_{j}}{\mu_{0}}\right)\left|x_{j}\right\rangle .
$$

\footnotetext{
${ }^{3}$ One of these cases give rise to degenerate roots, such that the solution (2.16) changes for $\psi(j)=r^{j}\left(a_{1}+a_{2} j\right)$.
} 


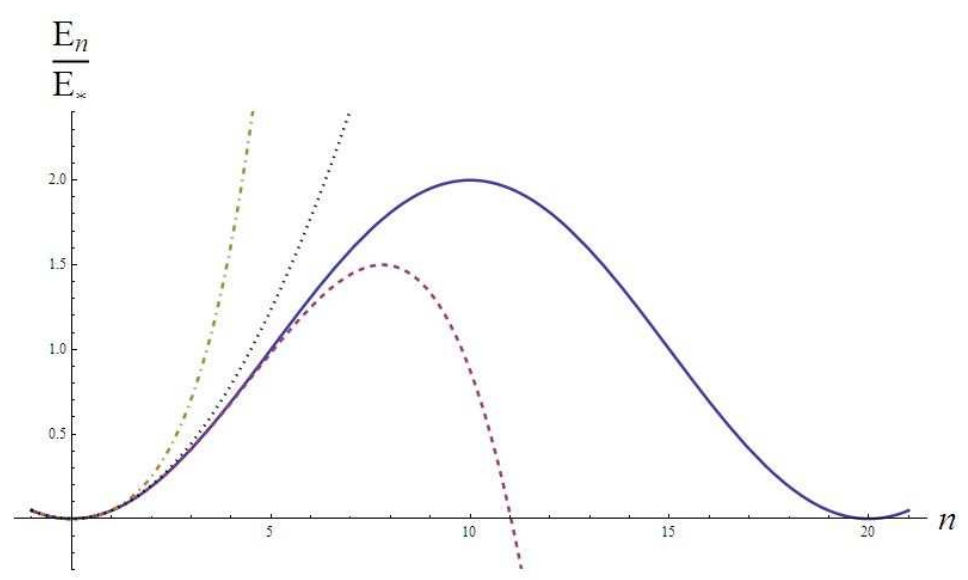

Figure 1. The solid line (blue) corresponds to the first Brillouin zone for the spectrum (2.21) and the dashed line (red) is the second order approximation equation (2.22). The dot-dashed line (green) is the case reported in the GUP literature corresponding to equation (2.23) which presents and opposite tendency with respect to the previous one. The dotted line (black) corresponds to the standard case $\lambda=0$.

We notice that the sum in (2.20) goes form $j=0, \ldots, N$. Since $n$ is an integer it is clear that we can not build the $n+1$ eigenstate because it would depend on the previous $n$ states. Thus $0<n<N$. The corresponding energy spectrum is found to be bounded [54]

$$
E_{n}=\frac{\hbar^{2}}{m \mu_{0}^{2}}(1-\cos n \pi \lambda), \quad n \in\{1,2, \ldots, N-1\}
$$

The energy spectrum (2.21) resembles the tight binding model for particles in a periodic potential that is not infinitely high and allow tunneling with the nearest neighbors sites $[55,56,57]$ :

$$
E(\kappa)=E_{*}-2 \Delta \cos \kappa \mu_{0},
$$

where $E_{*}=\frac{\hbar^{2}}{m \mu_{0}^{2}}$ and $\Delta$ represents the relevant non diagonal terms that give rise to the energy band, $\kappa$ is interpreted as a wave vector which take values on $\left[-\frac{\pi}{\mu_{0}}, \frac{\pi}{\mu_{0}}\right]$, and which, by choosing periodic boundary conditions [56,57], it takes discrete values $\kappa=\frac{\pi m}{L}$ with $N \in \mathbb{Z}$ and $-N \leq$ $m \leq N$.

Hence, the polymer particle in a box is analogous to the tight binding model of a particle in a periodic potential with periodic boundary conditions, having band energy $\Delta=E_{*} / 2$. So there is an energy band appearing in this polymer system given by a energy spectrum bounded from above and below, and a kind of Brillouin zone as shown in Fig. 1.

If we expand $\cos n \pi \lambda$, for $\lambda \ll 1$, i.e. large size of the box as compared to the lattice spacing, up to second order, we get

$$
E_{n}=\frac{\hbar^{2} n^{2} \pi^{2}}{2 m L^{2}}-\frac{\hbar^{2} n^{4} \pi^{4}}{24 m L^{2}} \lambda^{2}+\cdots
$$

Using $\mu_{0}=10^{-19} \mathrm{~m}$ and with the approximated spectrum (2.22), the correction will be significant only when $n \approx 10^{17}$.

The energy spectrum of a quantum particle in a box has also been studied in the framework of a GUP [58]. In this frame the modifications induced by a minimum length $\ell_{\min }$ on the wave function and the energy spectrum of a particle in a one-dimensional box, result in a modification 
of the Schrödinger equation which transforms it in a fourth order differential equation. The energy spectrum has a correction proportional to the squared of the minimum length $\ell_{\min }^{2}$

$$
E_{n}^{(\mathrm{GUP})}=\frac{n^{2} \pi^{2} \hbar^{2}}{2 m L^{2}}+\frac{\ell_{\min }^{2}}{L^{2}} \frac{n^{4} \pi^{4} \hbar^{2}}{3 m L^{2}} .
$$

This result can be compared with (2.22). We can see that the dependence on the minimum scale is quadratic in both cases but they feature a sign difference thus signaling opposite tendencies. As we already mentioned this coincidence is not surprising since polymer systems have similar modifications to GUP in their corresponding uncertainty relation [39]. However, in [58] the usual boundary condition for a particle in a box is used; here, since the measurement of the position has some uncertainty proportional to the minimum length, the position of the walls is not determined precisely. This problem is solved recalling that in polymer quantum mechanics the dynamics is defined on superselection spaces for which there is, in each one, a proper boundary conditions defined by (2.13) and (2.14).

\section{Polymer corrections to thermodynamic quantities of simple systems}

In standard statistical mechanics [59], the canonical partition function $Z$ is defined as the sum over all possible states

$$
Z(\beta)=\sum_{n} \exp \left(-\beta E_{n}\right)
$$

where $\beta=(k T)^{-1}, k$ is the Boltzmann's constant, and $T$ is the temperature. With it we can calculate all thermodynamical quantities of the system, through the definition of the Helmholtz free energy [59]

$$
F=-\frac{\mathcal{N}}{\beta} \ln Z,
$$

where $\mathcal{N}$ is the particle number. The relationship with other thermodynamic quantities such as the equation of state, entropy and chemical potential can be obtained by the standard relations

$$
\begin{aligned}
p & =-\frac{\partial F}{\partial L}, \\
\mu & =\frac{\partial F}{\partial \mathcal{N}}, \\
S & =k \beta^{2} \frac{\partial F}{\partial \beta} .
\end{aligned}
$$

Moreover the internal energy and the heat capacity can also be related to the partition function as relations

$$
\begin{aligned}
& U=-\mathcal{N} \frac{\partial \ln Z}{\partial \beta}=-\frac{\mathcal{N}}{Z} \frac{\partial Z}{\partial \beta}, \\
& C_{V}=-k \beta^{2} \frac{\partial U}{\partial \beta} .
\end{aligned}
$$

Thus, all we need is to calculate the partition function (3.1) using the corresponding energy spectrum.

Here we use both closed forms (2.8), (2.9) and (2.21), as well as the approximate forms (2.10) and (2.22) of the spectra to calculate the canonical partition function (3.1) and to obtain thermodynamical quantities for the polymer solid and ideal gas. 
In this work we have adopted the Maxwell-Boltzmann statistics for particles with no spin. However, it has been argued that modified statistics may be needed in quantum gravity and discrete theories. The relation between the discrete structure and statistics is still open [60,61].

\subsection{Ensemble of polymer oscillators}

\subsubsection{Exact spectrum}

To calculate the partition function using the spectrum (2.8), (2.9), we split the sum into two parts

$$
Z(\beta)=\sum_{n} \exp \left[-\frac{\beta \hbar \omega}{\lambda^{2}}\left(1+\frac{\lambda^{4}}{8} a_{n}\left(\frac{4}{\lambda^{4}}\right)\right)\right]+\sum_{n^{\prime}} \exp \left[-\frac{\beta \hbar \omega}{\lambda^{2}}\left(1+\frac{\lambda^{4}}{8} b_{n^{\prime}+1}\left(\frac{4}{\lambda^{4}}\right)\right)\right],
$$

the first term takes into account the even, and the second term the odd, parts of the spectrum with $n$ and $n^{\prime}$ running over all integers.

To determine the thermodynamical quantities we first write the Helmoltz free energy as

$$
F=-\frac{\mathcal{N}}{\beta} \ln \left\{e^{-\frac{\beta \hbar \omega}{\lambda^{2}}} \sum_{n}\left[\exp \left(-\beta \hbar \omega \frac{\lambda^{2}}{8} a_{n}\right)+\exp \left(-\beta \hbar \omega \frac{\lambda^{2}}{8} b_{n+1}\right)\right]\right\},
$$

where we omit the argument $\frac{4}{\lambda^{4}}$ of the characteristic Mathieu functions to avoid cumbersome expressions. From (3.3) and (3.4) it can be noticed that the equation of state and the chemical potential remain unchanged with respect to the standard case. On one hand, the Helmholtz free energy does not depend on the length of the system, and, on the other, its dependence on $\mathcal{N}$ is not modified with respect to the standard case.

The expressions for the entropy, internal energy and heat capacity are the following:

$$
\begin{aligned}
& \frac{S}{\mathcal{N} k}= \ln \left\{e^{-\frac{\beta \hbar \omega}{\lambda^{2}}} \sum_{n}\left[e^{-\beta \hbar \omega \frac{\lambda^{2}}{8} a_{n}}+e^{-\beta \hbar \omega \frac{\lambda^{2}}{8} b_{n+1}}\right]\right\} \\
&+\frac{\beta \hbar \omega}{Z} \frac{e^{-\frac{\beta \hbar \omega}{\lambda^{2}}}}{\lambda^{2}} \sum_{n}\left[A_{n} e^{-\beta \hbar \omega \frac{\lambda^{2}}{8} a_{n}}+B_{n} e^{-\beta \hbar \omega \frac{\lambda^{2}}{8} b_{n+1}}\right], \\
& U=\frac{\mathcal{N} \hbar \omega}{Z} \frac{e^{-\frac{\beta \hbar \omega}{\lambda^{2}}}}{\lambda^{2}} \sum_{n}\left[A_{n} e^{-\beta \hbar \omega \frac{\lambda^{2}}{8} a_{n}}+B_{n} e^{-\beta \hbar \omega \frac{\lambda^{2}}{8} b_{n+1}}\right], \\
&\left.\frac{C_{V}}{\mathcal{N} k}=\frac{e^{-\beta \hbar \omega \frac{1}{\lambda^{2}}} \frac{(\beta \hbar \omega)^{2}}{Z}\left\{\sum _ { n } \left[A_{n} e^{-\beta \hbar \omega \frac{\lambda^{2}}{8} a_{n}}\left(A_{n}-\frac{2 \lambda^{2}}{\beta \hbar \omega}\right)\right.\right.}{}+B_{n} e^{-\beta \hbar \omega \frac{\lambda^{2}}{8} b_{n+1}}\left(B_{n}-\frac{2 \lambda^{2}}{\beta \hbar \omega}\right)\right]-\frac{e^{-\frac{\beta \hbar \omega}{\lambda^{2}}}}{Z}\left\{\sum_{n}\left[A_{n} e^{-\beta \hbar \omega \frac{\lambda^{2}}{8} a_{n}}+B_{n} e^{-\beta \hbar \omega \frac{\lambda^{2}}{8} b_{n+1}}\right]\right\}^{2} \\
&\left.+\frac{2 \lambda^{2}}{\beta \hbar \omega} \sum_{n}\left[A_{n} e^{-\beta \hbar \omega \frac{\lambda^{2}}{8} a_{n}}+B_{n} e^{-\beta \hbar \omega \frac{\lambda^{2}}{8} b_{n+1}}\right]\right\},
\end{aligned}
$$

where

$$
A_{n} \equiv\left(\frac{\lambda^{4}}{8} a_{n}+1\right), \quad B_{n} \equiv\left(\frac{\lambda^{4}}{8} b_{n+1}+1\right) .
$$

These sums can not be reduced to a simple form but they can be treated numerically. These thermodynamical functions for arbitrary $\lambda$ differ significatively with respect to the standard 


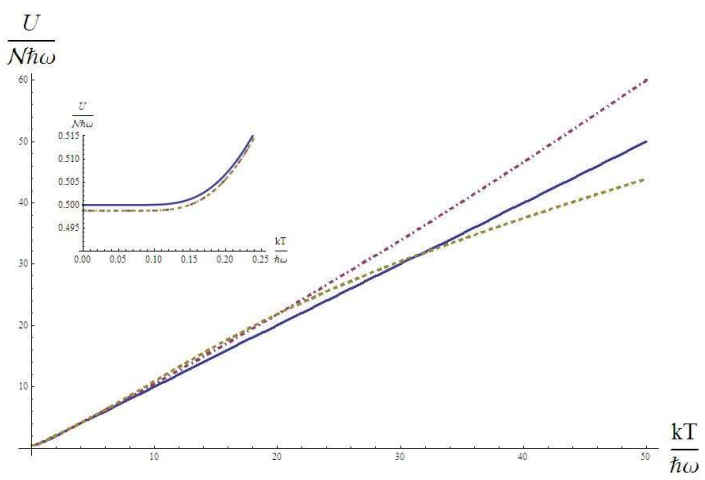

(a)

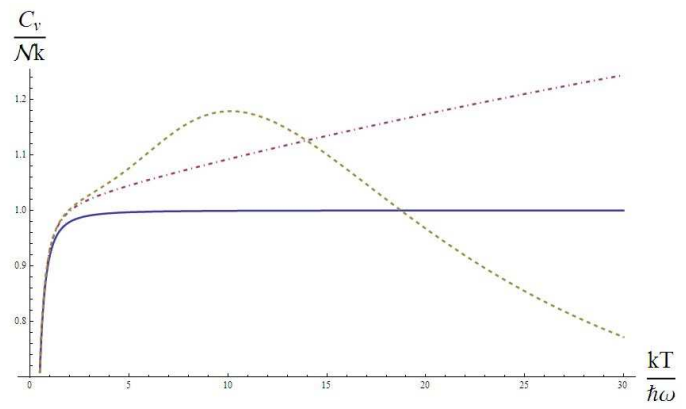

(b)

Figure 2. Here we plot the internal energy $U$ and the heat capacity as functions of $k T / \hbar \omega$. In both graphics the solid (blue) line corresponds to the standard case with $\lambda=0$, the dashed (green) lines corresponds to (3.9) and (3.10), respectively, for $\lambda=0.2$. The dotdashed (red) lines correspond to the approximated quantities (3.14) and (3.15) also for $\lambda=0.2$. For the internal energy $U$ we can see that the ground energy is shifted in the inbox of (a) to a lower value, for high temperatures the exact modified behavior decreases, while the approximated one increases with respect to the standard case. For the heat capacity $C_{V}$ we notice that for low temperature the three cases are very similar (b), while for high temperatures the behavior is completely different.

case (see Fig. 2). However, for small $\lambda$ the polymer and standard cases behave qualitatively in a similar manner.

Next we study the afore mentioned limiting cases. The case $\lambda \ll 1$, corresponds to small deviations from the standard case $\lambda=0$. Although the case $\lambda \gg 1$ has no direct interpretation in terms of length scales, we can say that it corresponds to the case when the energy of the system is much greater than the energy associated with the polymer scale.

\subsubsection{Approximate spectrum $\lambda \ll 1$ : ensemble of harmonic oscillators}

Using the approximation given in (2.10) for small $\lambda$, consistent with $n_{\max } \sim \lambda^{-2}$, the partition function becomes

$$
Z(\beta)=\sum_{n=0}^{\lambda^{-2}} e^{-\beta \hbar \omega\left(\frac{1}{2}+n\right)} \exp \left[\frac{\lambda^{2}}{16} \beta \hbar \omega\left(\frac{1}{2}+n(n+1)\right)\right],
$$

summations can be performed in a closed form to yield

$$
Z(\beta) \simeq \frac{e^{-\frac{\beta \hbar \omega}{2}}}{1-e^{-\beta \hbar \omega}}\left[1+\frac{\lambda^{2}}{32} \beta \hbar \omega\left(\frac{1+e^{-\beta \hbar \omega}}{1-e^{-\beta \hbar \omega}}\right)^{2}+\mathcal{O}\left(e^{-\frac{1}{\lambda^{2}}}\right)\right] .
$$

We recognize the first term in (3.11) as the usual partition function of the standard oscillator; the second term is the leading polymer correction and we are neglecting terms of order $\mathcal{O}\left(e^{-\frac{1}{\lambda^{2}}}\right)$ which clearly tend to zero in the limit $\lambda \rightarrow 0$.

The regime where the first term is much larger than corrections, i.e. the classical regime, corresponds to $\beta \hbar \omega \gg 1$. This can be seen in Fig. 2. The polymer oscillator approximates the standard oscillator for low temperatures.

Using the partition function (3.11) we calculate the corresponding thermodynamic quantities, from (3.2). Then we can write a modified Helmholtz free energy

$$
F=\frac{\mathcal{N}}{\beta}\left[\frac{\beta \hbar \omega}{2}+\ln \left(1-e^{-\beta \hbar \omega}\right)-\ln \left(1+\lambda^{2} \frac{\beta \hbar \omega}{32}\left(\frac{1+e^{-\beta \hbar \omega}}{1-e^{-\beta \hbar \omega}}\right)^{2}\right)\right]
$$




$$
\cong \frac{\mathcal{N}}{\beta}\left[\frac{\beta \hbar \omega}{2}+\ln \left(1-e^{-\beta \hbar \omega}\right)-\lambda^{2} \frac{\beta \hbar \omega}{32}\left(\frac{1+e^{-\beta \hbar \omega}}{1-e^{-\beta \hbar \omega}}\right)^{2}\right] .
$$

Substituting (3.12) into (3.4), (3.5) we obtain: $\mu=F / \mathcal{N}$ and $p=0$ : i.e. the equation of state does not change. As for the entropy we have

$$
S=\mathcal{N} k\left[\frac{\beta \hbar \omega}{e^{\beta \hbar \omega}-1}-\ln \left(1-e^{-\beta \hbar \omega}\right)+\frac{(\lambda \beta \hbar \omega)^{2}}{8} e^{\beta \hbar \omega} \frac{e^{\beta \hbar \omega}+1}{\left(e^{\beta \hbar \omega}-1\right)^{3}}\right] .
$$

For the internal energy we found

$$
U=\mathcal{N} \hbar \omega\left[\frac{1}{2}+\frac{1}{e^{\beta \hbar \omega}-1}-\frac{\lambda^{2}}{32} \frac{\left(1+e^{\beta \hbar \omega}\right)\left(e^{2 \beta \hbar \omega}-4 e^{\beta \hbar \omega} \beta \hbar \omega-1\right)}{\left(e^{\beta \hbar \omega}-1\right)^{3}}\right],
$$

and for the heat capacity

$$
C_{V}=\mathcal{N} k(\beta \hbar \omega)^{2} \frac{e^{\beta \hbar \omega}}{\left(e^{\beta \hbar \omega}-1\right)^{2}}\left[1+\frac{\lambda^{2}}{8}\left(\frac{2+\beta \hbar \omega\left(1+4 e^{\beta \hbar \omega}\right)+e^{2 \beta \hbar \omega}(\beta \hbar \omega-2)}{\left(e^{\beta \hbar \omega}-1\right)^{2}}\right)\right] .
$$

We can see from (3.15) that heat capacity is increased due to the polymer correction, while it decreases the energy (3.14). Since $\beta \hbar \omega \gg 1$, such modifications are very small.

Physically, an ensemble of harmonic oscillators can be used to model vibrations in solids (phonons). In a solid the vibrational modes are modeled as a collection of harmonic oscillators in the so-called harmonic approximation which consists of approximating the classical interaction Hamiltonian of the atoms in a solid by a second order Taylor series [59]. The simplest model is called the Einstein model which assumes that all vibrational modes have the same frequency $\omega$, and that the oscillators are independent with no interaction. The thermodynamic magnitudes (3.12), (3.13), (3.14) and (3.15) contain modifications to the thermodynamics of an Einstein solid by defining the vibrational temperature $\Theta_{V}=\hbar \omega / k$. Note that in this case the polymer scale is not involved in the definition of vibrational temperature.

\subsubsection{Approximate spectrum $\lambda \gg 1$ : ensemble of rotors}

Finally we consider the $\lambda \gg 1$ case for which the partition function becomes

$$
Z(\beta) \cong e^{-\frac{\beta \hbar \omega}{\lambda^{2}}}+e^{-\frac{\beta \hbar \omega}{\lambda^{2}}} \sum_{n=1}^{\infty} 2 e^{-\frac{\beta \hbar \omega}{8} \lambda^{2} n^{2}}=e^{-\frac{\beta \hbar \omega}{\lambda^{2}}} \vartheta_{3}\left(0, e^{-\frac{\beta \hbar \omega}{8} \lambda^{2}}\right),
$$

where $\vartheta_{3}$ is the Jacobi's elliptic theta function [49]. As in previous cases, chemical potential and pressure are unaffected, while entropy internal energy and heat capacity are as follows:

$$
\begin{aligned}
& F=-\frac{\mathcal{N}}{\beta} \ln \left(e^{-\frac{\beta \hbar \omega}{\lambda^{2}}} \vartheta_{3}\right), \\
& \frac{S}{\mathcal{N} k}=\ln \left(e^{-\frac{\beta \hbar \omega}{\lambda^{2}}} \vartheta_{3}\right)+\frac{\lambda^{2} \beta \hbar \omega}{\vartheta_{3}}\left[\frac{e^{-\frac{\lambda^{2} \beta \hbar \omega}{8}} \vartheta_{3}^{\prime}}{8}+\frac{\vartheta_{3}}{\lambda^{4}}\right], \\
& U=\mathcal{N} \hbar \omega \frac{\lambda^{2}}{\vartheta_{3}}\left[\frac{e^{-\frac{\lambda^{2} \beta \hbar \omega}{8}} \vartheta_{3}^{\prime}}{8}+\frac{\vartheta_{3}}{\lambda^{4}}\right], \\
& \frac{C_{V}}{\mathcal{N} k}=\frac{(\beta \hbar \omega)^{2}}{64 \lambda^{2}}\left[\frac{e^{-\frac{\lambda^{2} \beta \hbar \omega}{4}}}{\vartheta_{3}^{2}}\left(\lambda^{6}\left(\vartheta_{3} \vartheta_{3}^{\prime \prime}-\vartheta_{3}^{\prime 2}\right)+e^{\frac{\lambda^{2} \beta \hbar \omega}{8}}\left(\lambda^{2}-\frac{16}{\beta \hbar \omega}\right) \vartheta_{3} \vartheta_{3}^{\prime} \lambda^{4}\right)-\frac{128}{\beta \hbar \omega}\right]
\end{aligned}
$$




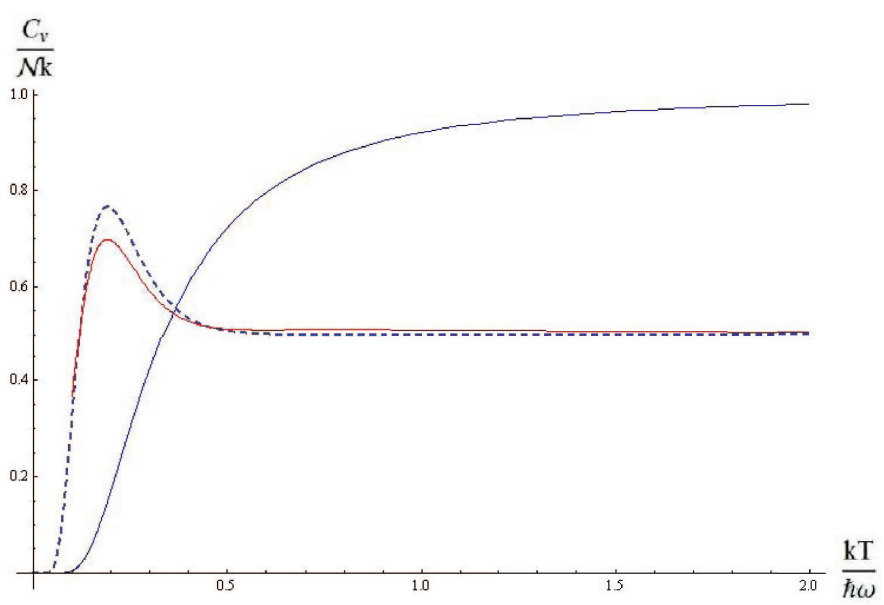

Figure 3. $C_{V}$ for the polymer oscillator. Blue line corresponds to the standard case with $\lambda=0$, dashed line is the approximation using the partition function (3.16) for $\lambda \gg 1$, and red line corresponds to the case using the full spectrum in terms of Mathieu characteristic functions in the same regime. We observe that, except for the value of the maximum of $C_{V}$, the approximation is very good for most temperatures.

$$
+\frac{2 \beta \hbar \omega \lambda^{2}}{\vartheta_{3}}\left[\frac{e^{-\frac{\lambda^{2} \beta \hbar \omega}{8}} \vartheta_{3}^{\prime}}{8}+\frac{\vartheta_{3}}{\lambda^{4}}\right]
$$

Again we omitted the arguments 0 and $e^{-\frac{\beta \hbar \omega}{8} \lambda^{2}}$ of $\vartheta_{3}$, while the primes in $\vartheta_{3}^{\prime}$ and $\vartheta_{3}^{\prime \prime}$, correspond to the first and second derivatives of $\vartheta_{3}$ with respect to its second argument $e^{-\frac{\beta \hbar \omega}{8} \lambda^{2}}$. Notice from Fig. 3, that the heat capacity of this system tends asymptotically to its classical value at high temperatures, while for low temperatures tends to zero, before passing through a maximum. This same behavior is found in the rotational contribution of particles with internal structure or an ensemble of rotors [59]. Hence for large $\lambda$ the quantum polymer oscillator approximates the rotor.

Actually, one can define the corresponding rotational temperature for this system. In the standard case the rotational temperature is $\Theta_{r} \equiv \frac{\hbar^{2}}{2 I k}$, with $I$ the moment of inertia. Using in this case the effective moment of inertia $I_{\text {eff }}=\frac{4 \hbar}{\omega \lambda^{2}}$, the rotational temperature turns out to be $\Theta_{r} \equiv \frac{\hbar \omega \lambda^{2}}{8 k}=\frac{m \omega^{2} \mu_{0}^{2}}{8 k}$, which does depend on $\lambda$. Note that the vibrational and rotational temperatures for the system differ by a factor $\Theta_{r} / \Theta_{V}=\lambda^{2} / 8$, which indicates that for small $\lambda$ the rotational states are negligible, while the oscillatory states are for large $\lambda$.

The full behavior of the polymer system can be seen in Fig. 2. The ensemble of polymer oscillators behave just as an ensemble of quantum pendulums. For low temperature it approaches the usual ensemble of oscillators, whereas at high temperatures it approaches an ensemble of rotors. This behavior is evident when studying the limiting cases separately as we have done here.

As usual in ensembles of systems from a spectrum containing $n^{2}$, there is a maximum in $C_{V}$ separating different behaviors. From energy considerations, this peak appears due to a change of concavity in $U$, which can be seen in Fig. 2(a). In the polymer oscillator as well as for the pendulum, the maximum in $C_{V}$ separates the rotational from the oscillatory behavior as a kind of smooth phase transition [62]. Given a fixed $\lambda$ and for low temperatures, the oscillatory states are turned on. As temperature increases more energetic states appear and for the value of temperature at which $C_{V}$ is maximum rotational states arise. For high temperatures, only the rotational states remain excited. Moreover, it should be noticed that the maximum of $C_{V}$ depends on $\lambda$. For $\lambda$ small enough, the temperature at which the maximum occur, would be very high so that the system approximates very well the oscillatory behavior, while to reach the rotational behavior one would need a huge amount of energy. 


\subsection{Partition function for the polymer ideal gas}

\subsubsection{Exact spectrum}

In the previous section we found that the energy spectrum of a polymer quantum particle in a box is proportional to $\cos n \pi \lambda$, namely equation (2.21). We also showed that if we expand $\cos n \pi \lambda$, when $\lambda \ll 1$, i.e., if the dimensions of the box are large compared to the minimum scale $\mu_{0}$, we obtain the usual quantum spectrum with modifications of order $\mathcal{O}\left(\lambda^{2}\right)$ equation $(2.21)$.

We can calculate the partition function with either the exact $(2.21)$ or approximated spectrum (2.22). Let us use the full spectrum (2.21). One way of introducing the approximation $n \lesssim \lambda^{-1}$, for which the analysis is valid, is to consider it as a cut-off in the energy, similarly as what has been done in [21]. First we rewrite the partition function as follows

$$
Z(\beta)=e^{-\frac{\Lambda^{2}}{2 \pi \mu_{0}^{2}}} \sum_{n=0}^{\infty} \exp \left(\frac{\Lambda^{2}}{2 \pi \mu_{0}^{2}} \cos n \pi \lambda\right)
$$

where $\Lambda=\sqrt{\frac{2 \pi \beta \hbar^{2}}{m}}$ is the thermal wave length [59], and the exponential in the argument of the sum can be written conveniently as an infinite sum of modified Bessel functions of first order [49]

$$
e^{\frac{\Lambda^{2}}{2 \pi \mu_{0}^{2}} \cos n \pi \lambda}=I_{0}\left(\frac{\Lambda^{2}}{2 \pi \mu_{0}^{2}}\right)+2 \sum_{k=1}^{\infty} I_{k}\left(\frac{\Lambda^{2}}{2 \pi \mu_{0}^{2}}\right) \cos (k n \pi \lambda) .
$$

Then we can replace (3.18) in (3.17) and perform the sum over $n$. However, we immediately see that the first term diverges. It is then necessary to consider the sum only up to $1 / \lambda$, as dictated by the approximation. Moreover, when $\lambda \rightarrow 0$, the limit of the sum tends to $\infty$, as in the standard case. Thus, the sum in (3.17) becomes

$$
\sum_{n=0}^{1 / \lambda} \exp \left(\frac{\Lambda^{2}}{2 \pi \mu_{0}^{2}} \cos n \pi \lambda\right)=\frac{1}{\lambda} I_{0}\left(\frac{\Lambda^{2}}{2 \pi \mu_{0}^{2}}\right)+\cosh \left(\frac{\Lambda^{2}}{2 \pi \mu_{0}^{2}}\right)+\sum_{k=1}^{\infty} I_{k}\left(\frac{\Lambda^{2}}{2 \pi \mu_{0}^{2}}\right) \cot \left(\frac{k \pi \lambda}{2}\right) \sin k \pi .
$$

In the last term $\cot k \pi \lambda / 2 \sim 2 /(k \pi \lambda)$ for small $\lambda$, then it is zero for any integer $k$. The partition function for this case has the form:

$$
Z(\beta)=I_{0}\left(\frac{\Lambda^{2}}{2 \pi \mu_{0}^{2}}\right) \frac{e^{-\frac{\Lambda^{2}}{2 \pi \mu_{0}^{2}}}}{\lambda}+\frac{1}{2}\left(1+e^{-\frac{\Lambda^{2}}{\pi \mu_{0}^{2}}}\right) .
$$

Let us notice that the last term $e^{-\frac{\Lambda^{2}}{\pi \mu_{0}^{2}}}$ tends to zero as $\mu_{0} \rightarrow 0$, also there is a constant term $1 / 2$, which only redefines the scale of $Z^{4}$. Consider then the partition function as

$$
Z(\beta)=I_{0}\left(\frac{\Lambda^{2}}{2 \pi \mu_{0}^{2}}\right) \frac{e^{-\frac{\Lambda^{2}}{2 \pi \mu_{0}^{2}}}}{\lambda},
$$

\footnotetext{
${ }^{4}$ Moreover, one can think that this term comes from the way we approximate the sum. We can realize this by using the Euler-Maclaurin formula to calculate the sum in the partition function, this calculation is usual in standard statistical mechanics

$$
\sum_{n=0}^{N} f(n) \cong \int_{0}^{N} f(n) d n+\frac{1}{2}(f(0)+f(N))+\cdots
$$

using $N=1 / \lambda$ and $f(n)=\exp \left(\frac{\Lambda^{2}}{2 \pi \mu_{0}^{2}} \cos n \pi \lambda\right)$ we find, as we shall see, that the partition function coincides with the asymptotic expansion of (3.19) and also contains the factor $1 / 2$, then it is not due to polymer corrections and we can ignore it from now on.
} 


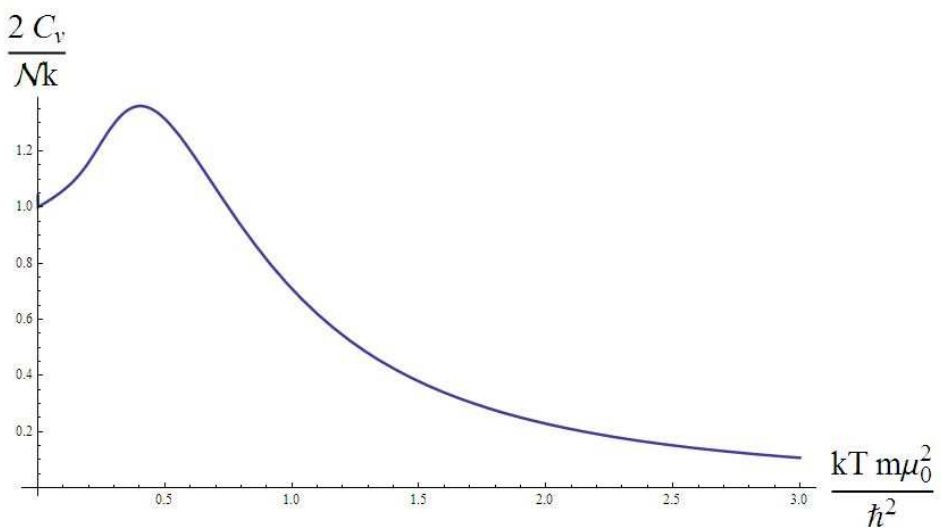

Figure 4. The $C_{V}$ for the polymer ideal gas has a maximum around $T \sim 2 \Theta_{\text {poly }}$, for smaller values of temperature one recovers the usual behavior, and when $T \rightarrow \infty, C_{V}$ tends to zero.

and let us calculate the corresponding thermodynamical quantities by recalling that the Helmholtz free energy in the case of indistinguishable particles can be determined by the relation $F=-\beta^{-1} \ln \left(\frac{Z^{\mathcal{N}}}{\mathcal{N} !}\right),[59]$, which in terms of $\beta$ reads

$$
F=-\frac{\mathcal{N}}{\beta}\left[1-\ln \mathcal{N}+\ln \left(\frac{L}{\mu_{0}} I_{0} e^{-\frac{\Lambda^{2}}{2 \pi \mu_{0}^{2}}}\right)\right],
$$

the other thermodynamical quantities are as follows:

$$
\begin{aligned}
& \mu=\frac{1}{\beta}\left[\ln \mathcal{N}-\ln \left(\frac{L}{\mu_{0}} I_{0} e^{-\frac{\Lambda^{2}}{2 \pi \mu_{0}^{2}}}\right)\right], \quad p=\frac{\mathcal{N}}{\beta L}, \\
& S=\mathcal{N} k\left[1-\ln \mathcal{N}+\ln \left(\frac{L}{\mu_{0}} I_{0} e^{-\frac{\Lambda^{2}}{2 \pi \mu_{0}^{2}}}\right)+\frac{\hbar^{2} \beta}{m \mu_{0}^{2}}\left(1-\frac{I_{1}}{I_{0}}\right)\right], \\
& U=\frac{\mathcal{N} \hbar^{2}}{m \mu_{0}^{2}}\left(1-\frac{I_{1}}{I_{0}}\right), \quad C_{V}=\frac{\mathcal{N} k}{2}\left(\frac{\hbar^{2} \beta}{m \mu_{0}^{2}}\right)^{2} \frac{I_{0}\left(I_{0}+I_{2}\right)-2 I_{1}^{2}}{I_{0}^{2}} .
\end{aligned}
$$

In all previous expressions we omit the arguments of the Bessel functions. We notice that also in this case, with the assumptions that we made, the equation of state remains unchanged.

From Fig. 4, we notice that for low temperature (with generic $\mu_{0}$ ) $C_{V}$ features the usual ideal gas behavior namely, it takes a constant value. On the other hand as temperature increases $C_{V}$ reaches a maximum (which depends on $\mu_{0}$ ) and then goes to zero as $T \rightarrow \infty$. This is consistent with the asymptotic behavior of the energy in the same regime. The maximum of $C_{V}$ occurs at about $T \sim \Theta_{\text {poly }} / 2$, where $\Theta_{\text {poly }} \equiv \frac{\hbar^{2}}{k m \mu_{0}^{2}}=\frac{E_{*}}{k}$. Remarkably, since the energy of the polymer particle in the box is bounded between 0 and $E_{*}$, we are just regaining the so called Schottky effect, [59], that appears for a two level quantum system in which the peak of $C_{V}$ appears for a temperature given by the energy difference between levels divided by $k$.

Moreover, it is well known that systems which are bounded from above, as the present case, allow the existence of negative temperatures [63]. From the thermodynamical definition of temperature, negative values of $T$ correspond to negative values in the slope of the graph of energy versus entropy. To see how this may happen one notices that in the partition function (3.1), and for positive temperatures, higher energy states contribute less than the low energy ones. this situation gets reversed for negative temperatures and in this situation it is mandatory that the energy be bounded above for the partition function to make sense. That's why only systems 


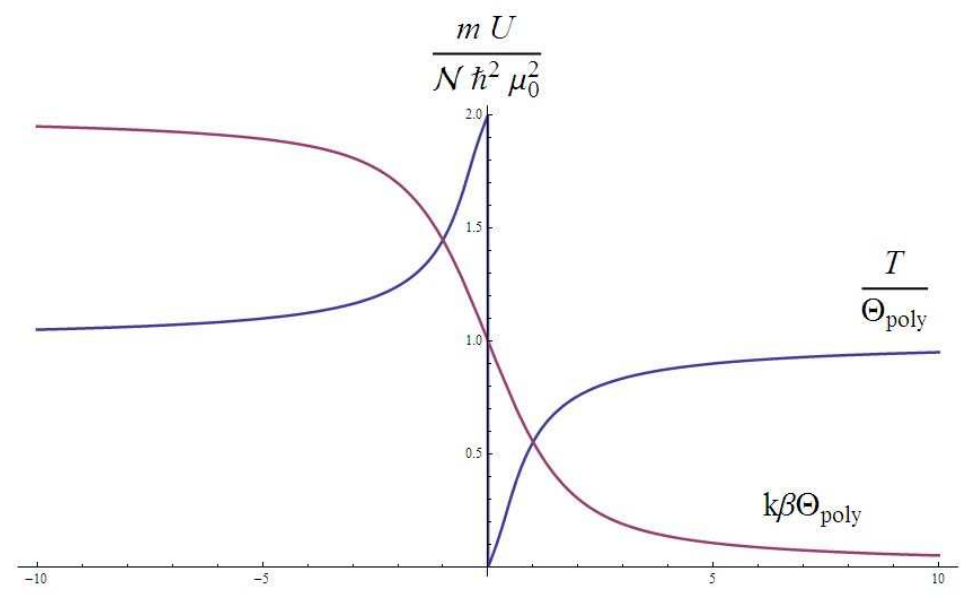

Figure 5. Internal energy of the polymer ideal gas as function of temperature $T$ and its reciprocal $\beta$ considering negative values of them.

such as those having two levels as magnetic systems and nuclear spin systems, feature negative temperatures [63]. Negative temperatures correspond to energies that are in principle experimentally accessible, due to the fact that $C_{V}$ remains positive for negative temperatures, e.g. in nuclear spin systems [63].

Now let us analyze our polymeric gas in the region of negative temperatures. In the limit $T \rightarrow 0^{+}, U$ reaches its minimum value which is zero; however, in the limit $T \rightarrow 0^{-}, U$ takes a value that is twice its asymptotic value. In the positive temperature region the energy increases from zero to its maximum value $\frac{\mathcal{N} \hbar^{2}}{m \mu_{0}^{2}}$, as $T \rightarrow \infty$. In the negative temperatures regime $U$ decreases from twice to once its asymptotic value as $T \rightarrow-\infty$. We can see this behavior from the Fig. 5. In the polymer case, when $\mu_{0}$ is very small, the maximum energy for positive temperature tends to infinity and we can not reach the negative temperature regime. To access negative temperature in the polymer case will be very difficult in the case $\lambda \ll 1$.

\subsubsection{Approximate spectrum}

Let us consider the limit $\lambda \rightarrow 0$ of the partition function (3.19) in order to regain the results for the standard ideal gas. We can make use of the asymptotic expansion of $I_{0}$ [49], which yields:

$$
Z(\beta) \approx \frac{L}{\Lambda}\left[1+\frac{\pi}{4} \frac{\mu_{0}^{2}}{\Lambda^{2}}+\frac{9 \pi^{2}}{32} \frac{\mu_{0}^{4}}{\Lambda^{4}}+\frac{75 \pi^{3}}{128} \frac{\mu_{0}^{6}}{\Lambda^{6}}+\frac{3675 \pi^{4}}{2048} \frac{\mu_{0}^{8}}{\Lambda^{8}}+\cdots\right]+\frac{1}{2}\left(1+e^{-\frac{\Lambda^{2}}{\pi \mu_{0}^{2}}}\right) .
$$

Of the two terms in round brackets in (3.20) only the $1 / 2$ remains in the $\mu_{0} \rightarrow 0$ limit. However, it only yields a constant shift in $Z$.

Interestingly, a different approximation from the above is to use the approximated spectrum (2.22) instead of (2.21) in the partition function. Since $\lambda \ll 1$ we consider only the first terms in the series of the second exponential, so that the partition function could be expressed as

$$
Z(\beta) \cong \sum_{n=0}^{\infty} e^{-\frac{\beta \hbar^{2} \pi^{2}}{2 m L^{2}} n^{2}}+\lambda^{2} \frac{\beta \hbar^{2} \pi^{4}}{24 m L^{2}} \sum_{n=0}^{\infty} n^{4} e^{-\frac{\beta \hbar^{2} \pi^{2}}{2 m L^{2}} n^{2}}+\mathcal{O}\left(\lambda^{4}\right) .
$$

Now, by the use of the Poisson resummation formula, namely

$$
\sum_{n=-\infty}^{\infty} f(n)=\sum_{n=-\infty}^{\infty} \int_{-\infty}^{\infty} f(y) e^{-2 \pi i y n} d y
$$


we can write the partition function as

$$
Z(\beta) \cong \sqrt{\frac{m L^{2}}{2 \pi \beta \hbar^{2}}}+\lambda^{2} \frac{\pi}{4}\left(\frac{m L^{2}}{2 \pi \beta \hbar^{2}}\right)^{3 / 2}+\cdots+\mathcal{O}\left(e^{-\frac{2 m L^{2}}{\beta \hbar^{2}}}\right),
$$

that is precisely the first term in (3.20). The first term is the standard partition function for the one dimensional ideal gas. This leads us to the constraint for the approximation to hold, that $\mu_{0} \ll \Lambda \sqrt{\frac{4}{\pi}}$.

Furthermore we can compute it with more orders in $\mu_{0}$

$$
Z(\beta) \cong \frac{L}{\Lambda}\left[1+\frac{\pi}{4} \frac{\mu_{0}^{2}}{\Lambda^{2}}+\frac{9 \pi^{2}}{32} \frac{\mu_{0}^{4}}{\Lambda^{4}}+\frac{75 \pi^{3}}{128} \frac{\mu_{0}^{6}}{\Lambda^{6}}+\frac{3675 \pi^{4}}{2048} \frac{\mu_{0}^{8}}{\Lambda^{8}}+\mathcal{O}\left(e^{-4 \pi \frac{L^{2}}{\Lambda^{2}}}, \mu_{0}^{10}\right)\right] .
$$

Let us use (3.22) to order $\mu_{0}^{2}$ to obtain the approximate thermodynamic quantities:

$$
F=\frac{-\mathcal{N}}{\beta}\left[1-\ln \mathcal{N}+\ln L-\frac{1}{2} \ln \beta+\ln \sqrt{\frac{m}{2 \pi \hbar^{2}}}+\ln \left(1+\frac{\mu_{0}^{2}}{8} \frac{m}{\beta \hbar^{2}}\right)\right] .
$$

We notice that $F$ diverges for $\beta=0$ or $T \rightarrow \infty$, but with the opposite sign than in the usual case. As we approach to $\beta=0$ we found a maximum after which the free energy diverges to $-\infty$ at $\beta=0$. This means that the effect of the minimum length scale on the free energy, is to provide a turning point for $F$ at very high temperatures. Furthermore, when we increase the value of $\mu_{0}$, the free energy becomes negative at high temperatures.

We can calculate gas pressure through the relationship $p=-\partial F / \partial L$, which is valid for one dimensional system. Realizing that the term that comes from the polymer correction does not depend on $L$, but on $\mu_{0}$, we can obtain the equation of state for the ideal gas as $p=N / \beta L$, which is the same as in the continuum case. The polymer corrections can not be seen as an effective interaction among the particles as it was the case for GUP or MDR [35].

Recalling (3.3) and (3.5) the chemical potential and the entropy of the gas can be calculated

$$
\begin{aligned}
& \mu=-\frac{1}{\beta}\left[-\ln \mathcal{N}+\ln L-\frac{1}{2} \ln \beta+\ln \sqrt{\frac{m}{2 \pi \hbar^{2}}}+\ln \left(1+\frac{\mu_{0}^{2}}{8} \frac{m}{\beta \hbar^{2}}\right)\right], \\
& S=\mathcal{N} k\left[\frac{3}{2}+\ln \frac{L}{\mathcal{N}}-\ln \beta^{1 / 2}+\ln \sqrt{\frac{m}{2 \pi \hbar^{2}}}+\frac{\mu_{0}^{2} m}{8 \hbar^{2} \beta}+\ln \left(1+\frac{\mu_{0}^{2} m}{8 \hbar^{2} \beta}\right)\right] .
\end{aligned}
$$

The last expression (3.23) would be the equivalent of a one dimensional Sackur-Tetrode's formula for the entropy with corrections due to the underlying discreteness. The energy and heat capacity are obtained from (3.6) and (3.7) respectively as

$$
\begin{aligned}
& U=\frac{\mathcal{N}}{2 \beta}\left(1+\frac{\mu_{0}^{2}}{4} \frac{m}{\hbar^{2} \beta}+\cdots\right), \\
& C_{V}=\frac{k \mathcal{N}}{2}\left(1+\frac{\mu_{0}^{2}}{2} \frac{m}{\hbar^{2} \beta}+\cdots\right) .
\end{aligned}
$$

All the above expressions are also obtained when considering the asymptotic expansion of Bessel functions of the quantities obtained in the previous section.

We notice from (3.24) that heat capacity has a different behavior with respect to the standard case for which $C_{V}$ is constant. For $\beta=0$, or high temperature, indeed it diverges in this approximate case. However, we know that this is only an approximation which corresponds, in the full case, to increasing $C_{V}$ to its maximum. Because heat capacity is related to energy fluctuations, we can say that for high temperatures there are strong fluctuations in the energy due to space discreteness. 
Notice that if we use the result (2.23) the calculations of the thermodynamical quantities remain the same and only differ by numerical factors, i.e. in the partition function (3.21) a factor $1 / 24$ is replaced by $-1 / 3$, etc. The main difference is that with (2.23) the thermodynamical quantities will decrease instead of increase as our results show.

\section{Discussion}

Polymer quantum mechanics considers mechanical models quantized in a similar way as loop quantum gravity but in which loops/graphs resembling polymers are replaced by discrete sets of points. It has allowed to study some features of loop quantum gravity in a simpler context, namely through the use of mechanical systems [19, 20]. Indeed this opened up the possibilities to investigate different physical problems [19, 26, 39, 27, 28]. On the other hand important gravitational systems like the cosmos itself and black holes necessarily involve thermodynamics in their description. However, little attention has been given to the thermostatistics of polymer quantum systems. In this work we embarked on this task using the canonical ensemble theory applied to a polymer solid and a polymer gas, both in one dimension. The resulting thermodynamic quantities have modifications due to the minimum length scale that introduces the polymer quantization. Thermodynamics with modifications due to quantum gravity has been studied in the context of extensions of the standard model with Lorentz violations [36] and also models that mimic the graphic states of loop representation, but which, however, do not represent physical situations [64]. It is important to stress that here the canonical partition function is used as the simplest case, the relation between the discrete space and the statistics is an open issue $[60,61]$.

First we consider an ensemble of polymer oscillators, and we noticed that it can be interpreted as an ensemble of quantum pendulums with two regimes: For small $\lambda$ one has the vibrational or simple oscillator regime, and for large $\lambda$ the rotational regime. Both behaviors are separated

by a maximum in the $C_{V}$. In the generic $\lambda$ case, we observe that to access the rotational regime requires a high temperature that also depends on $\lambda$. In that case the partition function and therefore the thermodynamic quantities, are written in terms of certain sums of the exponentials containing the Mathieu characteristics functions (3.8).

In the vibrational regime $\lambda \ll 1$ the partition function can be expressed as a power series on the minimum polymer length scale. Interestingly the equation of state is not modified. As is well known, with this model one can model an Einstein's solid introducing the vibrational temperature $\Theta_{V}$ that takes into account the characteristic energy of the system in this regime. We recover the known thermodynamical quantities when $\lambda \rightarrow 0$.

The rotational regime $\lambda \gg 1$ that corresponds to an ensemble of rotors, can be characterized by the rotational temperature $\Theta_{r}$ that in this case depends on $\lambda$, as $\Theta_{r}=\frac{\hbar \omega}{8 k} \lambda^{2}$. In this case $Z$ and the thermodynamical variables can be written in terms of the Jacobi's theta function $\vartheta_{3},(3.16)$. This regime can be of interest for the polymer analogous to the trans-planckian problem, since this case corresponds to energies beyond the characteristic polymer energy given by the coefficient of equation (2.5).

Amusingly it is possible to consider the effect of the polymer quantization in the case of electromagnetic radiation as follows. Let us recall that an ensemble of oscillators can be used to model excitations in solids (phonons), but also quantum excitations of the electromagnetic field (photons). Historically, equilibrium radiation was first studied by Planck in 1900, who considered this system as an ensemble of harmonic oscillators with the same frequency. Nowadays we consider that photons are ultra relativistic bosons with some particular energy $\hbar \omega$ [59]. Let us use Planck's simple model. By interpreting the internal energy as $\mathcal{N}$ times the average energy of each oscillator, i.e. $U=\mathcal{N}\left\langle E_{n}\right\rangle$, from which it follows that $\left\langle E_{n}\right\rangle=\frac{\hbar \omega}{2}+\langle n\rangle$, where $\langle n\rangle$ is the average occupation number that can be obtained from the previous equation and (3.14). This 
yields

$$
\langle n\rangle=\frac{1}{e^{\beta \hbar \omega}-1}-\frac{\lambda^{2}}{32} \frac{\left(1+e^{\beta \hbar \omega}\right)\left(e^{2 \beta \hbar \omega}-4 \beta \hbar \omega e^{\beta \hbar \omega}-1\right)}{\left(e^{\beta \hbar \omega}-1\right)^{3}},
$$

where the second term is the polymer correction. With (4.1) we can calculate the spectral density that is defined as

$$
u_{\omega} \equiv \frac{d}{d \omega}\left(\frac{U}{V}\right)=\langle n\rangle \hbar \omega \frac{g(\omega)}{V},
$$

where the density of states $g(\omega)=\frac{V \omega^{2}}{\pi^{2} c^{3}}, V$ being the volume. The expression (4.2) is thus the black body distribution now containing polymer corrections. Note that the spectral density for a fixed temperature is modified for high frequencies. We know that the cosmic background radiation $\mathrm{CMB}$ with $T \cong 3 K$ is the black body that has been measured more accurately [65]. This further constrains the possible value of $\lambda$ and therefore the minimum scale of $\mu_{0}$. In this case such changes do not alter the functional dependence on temperature as in other approaches $[34,35,37]$. Using (4.1) and (4.2) we can obtain directly the energy density by integration. It follows that the Stefan-Bolztmann law takes the form

$$
\frac{U}{V}=\frac{\pi^{2} k^{4}}{15 c^{3} \hbar^{3}}\left(1+\lambda^{2} \frac{135}{4 \pi^{4}} \zeta(3)\right) T^{4} \simeq \frac{\pi^{2} k^{4}}{15 c^{3} \hbar^{3}}\left(1+0.416485 \lambda^{2}\right) T^{4} .
$$

Thus, given (4.3) the Stefan-Boltzmann constant is modified by a term of order $\mathcal{O}\left(\lambda^{2}\right)$ as

$$
\sigma_{S B}^{(\lambda)}=\frac{\pi^{2} k^{4}}{60 c^{2} \hbar^{3}}\left(1+0.416485 \lambda^{2}\right) .
$$

where $\lambda$ is small. Notice that, as expected, we recover the usual formulae of thermodynamics of black body radiation for $\lambda \rightarrow 0$. Similar analysis using other proposal have been given in $[34,35,37]$.

As for the ideal polymer gas, the partition function was determined in two regimes depending on whether the spectrum is considered in its exact or approximate form. In the exact spectrum case we notice there is a cut-off in the energy levels proportional to $1 / \lambda$. This ensures in particular the convergence of the partition function (3.17). We note that in this system is quite evident that negative temperatures are allowed. In the approximate spectrum case we express the partition function as a series in powers of $\mu_{0}$. Of course standard thermodynamics is contained in our results in the limit $\lambda \rightarrow 0$. These results resemble those obtained from GUP $[32,58]$ in the sense they correspond to quadratic corrections although they feature opposite tendencies [44]. Clearly within our analysis the origin of the modifications can be traced all the way back to the polymer model, in which there is also a modified uncertainty relation [39].

In regard to the thermodynamics of both of our systems it is worth stressing a common behavior of their heat capacity. For the gas, in contrast to the standard case, the $C_{V}$ has a maximum of about half of $\Theta_{\text {poly }}$, the temperature at which the polymer effects are evident. The polymer oscillator, on the other hand, also shows a similar behavior in its $C_{V}$, having a maximum that separates two different behaviors characterized by $\Theta_{V}$ and $\Theta_{r}$.

Now we mention some possible extensions of this work. The canonical distribution adopted here is only intended to be an approximation. The statistics of polymer systems, which are naturally discrete is still an open issue [60]. In [61] the problem of calculating the number of accessible microstates was considered in a semiclassical perspective; it was found that the standard methods yield only approximate results. To count states in polymer systems and to include fermions and bosons, again a better understanding of the statistics of polymer quantum systems is needed. 
In this work we learnt that polymer quantization induces some effects on the statistical description of the systems, which in principle we can explore. This effects are such as the maximum in $C_{V}$ between two different behaviors, or the appearance of negative temperatures in the case of ideal gas. Interestingly, a similar behavior has been observed in gravitational systems such as black holes, where the heat capacity shows a phase transition that depends on the minimum length scale [66]. The study of the thermodynamic quantities of gravitational systems has focused mainly on obtaining the entropy of black holes from gravitational quantum states [67], or to find effective modifications to those thermodynamical quantities, [41, 68]. However, recently there has been some quantum gravity models which are based on thermostatistics of condensed matter systems, can yield interesting results [69, 70, 71].

Certainly an interesting question emerges when exploring the thermodynamics of non equilibrium processes such as those that occur in the early universe or in the black hole evaporation. Clearly it is necessary to extend the methods presented here to address these problems.

\section{Acknowledgements}

We would like to thank A. Camacho, M. Reuter, J.A. Zapata and E. Flores for useful discussions, comments and suggestions. We are indebted to Viqar Husain for communicating us recent results related to the present work [72]. Partial support from the following grants is acknowledged: CONACyT-NSF Strong backreaction effects in quantum cosmology, CONACyT 131138 and SNI-III research assistant 145852866 (GCA), DAAD A/07/95322 and CONACyT 55521 (EM).

\section{References}

[1] Rovelli C., Quantum gravity, Cambridge Monographs on Mathematical Physics, Cambridge University Press, Cambridge, 2004.

[2] Thiemann T., Modern canonical quantum general relativity, Cambridge Monographs on Mathematical Physics, Cambridge University Press, Cambridge, 2007.

[3] Bojowald M., Loop quantum cosmology, Living Rev. Relativity 8 (2005), 11, 99 pages, gr-qc/0601085.

[4] Bojowald M., Absence of a singularity in loop quantum cosmology, Phys. Rev. Lett. 86 (2001), 5227-5230, gr-qc/0102069.

[5] Bojowald M., Quantum nature of cosmological bounce, Gen. Relativity Gravitation 40 (2008), 2659-2683, arXiv:0801.4001.

[6] Ashtekar A., Pawlowski T., Singh P., Quantum nature of the big bang: improved dynamics, Phys. Rev. D 74 (2006), 084003, 23 pages, gr-qc/0607039.

[7] Rovelli C., Black hole entropy from loop quantum gravity, Phys. Rev. Lett. 77 (1996), 3288-3291, gr-qc/9603063.

[8] Ashtekar A., Baez J., Corichi A., Krasnov K., Quantum geometry and black hole entropy, Phys. Rev. Lett. 80 (1998), 904-907, gr-qc/9710007.

[9] Domagala M., Lewandowski J., Black-hole entropy from quantum geometry, Classical Quantum Gravity 21 (2004), 5233-5243, gr-qc/0407051.

[10] Kolb E.W., Turner M.S., The early universe, Paperback Ed. Westview Press, 1994.

[11] Weinberg S., Cosmology, Oxford University Press, Oxford, 2008.

[12] Grishchuk L.P., Sidorov Y.V., Relic graviton and the birth of the universe, Classical Quantum Gravity 6 (1989), L155-L160.

[13] Grishchuk L.P., Sidorov Y.V., Squeezed quantum states of relic gravitons and primordial density fluctuations, Phys. Rev. D 42 (1990), 3413-3421.

[14] Mielczarek J., Szydłowski M., Relic gravitons as the observable for loop quantum cosmology, Phys. Lett. B 657 (2007), 20-26, arXiv:0705.4449.

[15] Mielczarek J., Szydłowski M., Relic gravitons from super-inflation, arXiv:0710.2742. 
[16] Bojowald M., Hossain G.M., Loop quantum gravity corrections to gravitational wave dispersion, Phys. Rev. D 77 (2008), 023508, 14 pages, arXiv:0709.2365.

[17] Mielczarek J., Gravitational waves from the big bounce, J. Cosmol. Astropart. Phys. 11 (2008), 011, 17 pages, arXiv:0807.0712.

[18] Ashtekar A., Taveras V., Varadarajan M., Information is not lost in the evaporation of 2D black holes, Phys. Rev. Lett. 100 (2008), 211302, 4 pages, arXiv:0801.1811.

Ashtekar A., Pretorius F., Ramazanoğlu F.M., Evaporation of two-dimensional black holes, Phys. Rev. D 83 (2011), 044040, 18 pages, arXiv:1012.0077.

[19] Ashtekar A., Fairhurst S., Willis J.L., Quantum gravity, shadow states and quantum mechanics, Classical Quantum Gravity 20 (2003), 1031-1061, gr-qc/0207106.

[20] Corichi A., Vukašinac T., Zapata J.A., Polymer quantum mechanics and its continuum limit, Phys. Rev. D 76 (2007), 044016, 16 pages, arXiv:0704.0007.

[21] Corichi A., Vukašinac T., Zapata J.A., Hamiltonian and physical Hilbert space in polymer quantum mechanics, Classical Quantum Gravity 24 (2007), 1495-1511, gr-qc/0610072.

[22] Beaume R., Manuceau J., Pellet A., Sirugue M., Translation invariant states in quantum mechanics, Comm. Math. Phys. 38 (1974), 29-45.

Acerbi F., Morchio G., Strocchi F., Infrared singular fields and nonregular representations of canonical commutation relation algebras, J. Math. Phys. 34 (1993), 899-914.

Cavallaro F., Morchio G., Strocchi F., A generalization of the Stone-von Neumann theorem to nonregular representations of the CCR-algebra, Lett. Math. Phys. 47 (1999), 307-320.

Halvorson H., Complementarity of representations in quantum mechanics, Stud. Hist. Philos. Sci. B Stud. Hist. Philos. Modern Phys. 35 (2004), 45-56, quant-ph/0110102.

[23] Dobrev V., Doebner H.-D., Twarock R., Quantum mechanics with difference operators, Rep. Math. Phys. 50 (2002), 409-431, quant-ph/0207077.

[24] Fredenhagen K., Reszewski F., Polymer state approximation of Schrödinger wave functions, Classical Quantum Gravity 23 (2006), 6577-6584, gr-qc/0606090.

[25] Velhinho J.M., The quantum configuration space of loop quantum cosmology, Classical Quantum Gravity 24 (2007), 3745-3758, arXiv:0704.2397.

[26] Chiou D.-W., Galileo symmetries in polymer particle representation, Classical Quantum Gravity 24 (2007), 2603-2620, gr-qc/0612155.

[27] Husain V., Louko J., Winkler O., Quantum gravity and the Coulomb potential, Phys. Rev. D 76 (2007), 084002, 8 pages, arXiv:0707.0273.

[28] Kunstatter G., Louko J., Ziprick J., Polymer quantization, singularity resolution, and the $1 / r^{2}$ potential, Phys. Rev. A 79 (2009), 032104, 9 pages, arXiv:0809.5098.

[29] Amelino-Camelia G., Ellis J., Mavromatos N.E., Nanopoulos D.V., Sarkar S., Tests of quantum gravity from observations of $\gamma$-ray bursts, Nature 393 (1998), 763-765.

[30] Amelino-Camelia G., Introduction to quantum gravity phenomenology, in Planck Scale Effects in Astrophysics and Cosmology, Lecture Notes in Physics, Vol. 669, Springer-Verlag, Berlin, 2005, 59-100.

[31] Kalayana Rama S., Some consequences of the generalised uncertainty principle: statistical mechanical, cosmological, and varying speed of light, Phys. Lett. B 519 (2001), 103-110, hep-th/0107255.

[32] Nozari K., Mehdipour H., Implications of minimal length scale on the statistical mechanics of ideal gas, Chaos Solitons Fractals 32 (2007), 1637-1644, hep-th/0601096.

[33] Nozari K., Fazlpour B., Generalized uncertainty principle, modified dispersion relations and the early universe thermodynamics, Gen. Relativity Gravitation 38 (2006), 1661-1679, gr-qc/0601092.

[34] Nozari K., Sefidgar A.S., The effect of modified dispersion relations on the thermodynamics of black-body radiation, Chaos Solitons Fractals 38 (2008), 339-347.

[35] Camacho A., Macías A., Thermodynamics of a photon gas and deformed dispersion relations, Gen. Relativity Gravitation 39 (2007), 1175-1183, gr-qc/0702150.

[36] Colladay D., McDonald P., Statistical mechanics and Lorentz violation, Phys. Rev. D 70 (2004), 125007, 8 pages, hep-ph/0407354.

[37] Yépez H., Romero J.M., Zamora A., Corrections to the Planck's radiation law from loop quantum gravity, hep-th/0407072. 
[38] Alfaro J., Morales-Técotl H.A., Urrutia L.F., Loop quantum gravity and light propagation, Phys. Rev. D 65 (2002), 103509, 18 pages, hep-th/0108061.

[39] Hossain G.M., Husain V., Seahra S.S., Background-independent quantization and the uncertainty principle, Classical Quantum Gravity 27 (2010), 165013, 8 pages, arXiv:1003.2207.

[40] Reed M., Simon B., Methods of modern mathematical physics. I. Functional analysis, Academic Press Inc., New York, 1980.

[41] Peltola A., Kunstatter G., Effective polymer dynamics of D-dimensional black hole interiors, Phys. Rev. D 80 (2009), 044031, 13 pages, arXiv:0902.1746.

[42] Hossain G.M., Husain V., Seahra S.S., Nonsingular inflationary universe from polymer matter, Phys. Rev. D 81 (2010), 024005, 5 pages, arXiv:0906.2798.

[43] Kunstatter G., Louko J., Peltola A., Polymer quantization of the Einstein-Rosen wormhole throat, Phys. Rev. D 81 (2010), 024034, 10 pages, arXiv:0910.3625.

[44] Battisti M.V., Lecian O.M., Montani G., Polymer quantum dynamics of the Taub universe, Phys. Rev. D 78 (2008), 103514, 9 pages, arXiv:0806.0768.

[45] Battisti M.V., Lecian O.M., Montani G., GUP vs polymer quantum cosmology: the Taub model, arXiv:0903.3836.

[46] Creutz M., Quarks, gluons, and lattices, Cambridge University Press, Cambridge, 1983.

[47] Hossain G.M., Husain V., Seahra S., Propagator in polymer quantum field thepry, Phys. Rev. D 82 (2010), 124032, 5 pages.

[48] Chalbaud E., Gallinar J.-P., Mata G., The quantum harmonic oscillator on a lattice, J. Phys. A: Math. Gen. 19 (1986), L385-L390.

[49] Abramowitz M., Stegun I., Handbook of mathematical functions, Dover, 1968.

[50] Baker G.L., Blackburn J.A., Smith H.J.T., The quantum pendulum: small and large, Amer. J. Phys. 70 (2002), 525-531.

[51] Martin J., Brandenberger R.H., Trans-Planckian problem of inflationary cosmology, Phys. Rev. D 63 (2001), 123501, 16 pages, hep-th/0005209.

[52] Doncheski M.A., Robinett R.W., Wave packet revivals and the energy eigenvalue spectrum of the quantum pendulum, Ann. Physics 308 (2003), 578-598, quant-ph/0307079.

[53] Elaydi S., An introduction to difference equations, Undergraduate Texts in Mathematics, Springer-Verlag, New York, 1996.

[54] Gallinar J.P., Mattis D.C., Motion of 'hopping' particles in a constant force field, J. Phys. A: Math. Gen. 18 (1985), 2583-2589.

[55] Sakurai J.J., Modern quantum mechanics, Addison-Wesley, 1994.

[56] Marder M.P., Condenseed matter physics, John Wiley \& Sons, Inc., 2010.

[57] Kittel C., Introduction to solid state physics, John Wiley \& Sons, Inc., 1996.

[58] Nozari K., Azizi T., Some aspects of gravitational quantum mechanics, Gen. Relativity Gravitation 38 (2006), 735-742, quant-ph/0507018.

[59] Pathria R.K., Statistical mechanics, Butterworth-Heinemann, 2001.

[60] Swain J., Exotic statistics for ordinary particles in quantum gravity, Internat. J. Modern Phys. D 17 (2008), $2475-2484$.

[61] Chacón-Acosta G., Dagdug L., Morales-Técotl H., On microstates counting in many body polymer quantum systems, AIP Conf. Proc. 1396 (2011), 99-103.

[62] Naudts J., Boltzmann entropy and the microcanonical ensemble, Europhys. Lett. 69 (2005), 719-724, cond-mat/0412683.

[63] Ramsey N.F., Thermodynamics and statistical mechanics at negative absolute temperatures, Phys. Rev. 103 (1956), 20-28.

[64] Konopka T., Markopoulou F., Severini S., Quantum graphity: a model of emergent locality, Phys. Rev. D 77 (2008), 104029, 15 pages, arXiv:0801.0861.

Konopka T., Statistical mechanics of graphity models, Phys. Rev. D 78 (2008), 044032, 17 pages, arXiv:0805.2283. 
[65] Smoot G.F., Gorenstein M.V., Muller R.A., Detection of anisotropy in the cosmic blackbody radiation, Phys. Rev. Lett. 39 (1977), 898-901.

Fixsen D.J. et al., Cosmic microwave background dipole spectrum measured by the COBE FIRAS instrument, Astrophys. J. 420 (1994), 445-449.

Fixsen D.J. et al., The cosmic microwave background spectrum from the full COBE FIRAS data set, Astrophys. J. 473 (1996), 576-587, astro-ph/9605054.

[66] Husain V., Mann R.B., Thermodynamics and phases in quantum gravity, Classical Quantum Gravity 26 (2009), 075010, 6 pages, arXiv:0812.0399.

[67] Agullo I., Barbero G. J.F., Borja E.F., Diaz-Polo J., Villaseñor E.J.S., Detailed black hole state counting in loop quantum gravity, Phys. Rev. D 82 (2010), 084029, 31 pages, arXiv:1101.3660.

[68] Li L.-F., Zhu J.-Y., Thermodynamics in loop quantum cosmology, Adv. High Energy Phys. 2009 (2009), 905705, 9 pages, arXiv:0812.3544.

[69] Bianchi E., Black hole entropy, loop gravity, and polymer physics, Classical Quantum Gravity 28 (2011), 114006, 12 pages, arXiv:1011.5628.

[70] Hamma A., Markopoulou F., Background independent condensed matter models for quantum gravity, New J. Phys. 13 (2011), 095006, 26 pages, arxiv:1011.5754.

[71] Hamma A., Markopoulou F., Lloyd S., Caravelli F., Severini S., Markström K., Quantum Bose-Hubbard model with an evolving graph as a toy model for emergent spacetime, Phys. Rev. D 81 (2010), 104032, 22 pages, arXiv:0911.5075.

[72] Husain V., Seahra S., Webster E., in preparation.

Webster E., Oscillator gas in polymer quantum mechanics, Undergraduate Thesis, unpublished. 